\title{
Effects of dietary supplementation with EPA and vitamin E on the blood C-reactive protein content and antioxidant status of male basketball players
}

\author{
Abolghassem Djazayery, Reza Ghiasvand, Mahmood Djalali, Seeyed Ali Keshavarz \\ and Mostafa Hosseini \\ School of Public Health, Tehran University of Medical Sciences, Tehran, Islamic Republic of Iran
}

EPA, a very-long-chain $n-3$ fatty acid, has desirable health effects in healthy subjects and in some abnormal disease conditions ${ }^{(1)}$. There is also some evidence that $n-3$ fatty acids may have beneficial effects during exercise ${ }^{(2)}$, which may potentially be associated with oxidative stress. The objective of the present study was to investigate the effects of EPA supplementation, with or without vitamin E (an antioxidant vitamin), on the blood C-reactive protein (CRP) and total antioxidant capacity (TOAC) of male basketball players.

In a double-blind placebo-controlled clinical trial a total of thirty-two apparently-healthy well-trained 17-35-year-old male basketball players were divided into four groups of eight subjects who received by random allocation (/d): $2 \mathrm{~g}$ EPA (PlusEPA; MINAMI Nutrition, Edegem, Belgium) + a vitamin E placebo (EPA group); $400 \mathrm{mg}$ vitamin E (Zarovi Co., Tehran, Iran) + an EPA placebo (vitamin E group); $2 \mathrm{~g} \mathrm{EPA}+400 \mathrm{mg}$ vitamin E (EPA+ vitamin E group); vitamin E placebo + EPA placebo (placebo group). Venous blood samples at baseline (initial) and after 6 weeks (final) were obtained from all subjects between 17.00 hours and 18.00 hours after exercising for $2 \mathrm{~h}$. As the Table shows, CRP decreased in both the EPA and the EPA+ vitamin E groups, while TOAC increased only in the vitamin E group.

\begin{tabular}{|c|c|c|c|c|c|c|c|c|c|c|}
\hline & \multicolumn{5}{|c|}{ Plasma CRP (mg/l) } & \multicolumn{5}{|c|}{ TAOC $(\mathrm{nmol} / \mathrm{l})$} \\
\hline & \multicolumn{2}{|c|}{ Initial } & \multicolumn{2}{|c|}{ Final } & \multirow[b]{2}{*}{$P<$} & \multicolumn{2}{|c|}{ Initial } & \multicolumn{2}{|c|}{ Final } & \multirow[b]{2}{*}{$P<$} \\
\hline & Mean & SD & Mean & SD & & Mean & SD & Mean & SD & \\
\hline EPA & 28.5 & 9.6 & 12.2 & 4.2 & 0.001 & 30.1 & 2.9 & 30.0 & 2.2 & NS \\
\hline Vitamin E & 28.6 & 12.3 & 29.9 & 13.9 & NS & 29.0 & 2.7 & 34.1 & 1.5 & 0.002 \\
\hline $\mathrm{EPA}+$ vitamin $\mathrm{E}$ & 48.4 & 38.6 & 16.7 & 18.0 & 0.005 & 31.4 & 2.3 & 31.5 & 2.5 & NS \\
\hline Placebo & 22.9 & 10.7 & 24.3 & 12.7 & NS & 29.9 & 0.7 & 29.6 & 1.5 & NS \\
\hline
\end{tabular}

Inter-group comparisons at the end of the period revealed that the CRP content (mg/d) in the EPA group (12.2 (SD 4.2)) was lower than that in the vitamin E group (29.9 (SD 13.9); $P<0.05$ ). Furthermore, the TAOC (nmol/d) in the EPA group (30.0 (SD 2.2)) was lower than that in the vitamin E group (34.1 (SD 0.15); $P<0.001$ ), which was higher than the control (placebo) value (29.6 (SD 1.5); $P<0.001)$. It is concluded that while dietary supplementation with vitamin $\mathrm{E}$ alone has no effect on the plasma CRP level of the basketball players, it is decreased by EPA alone or the combination of PlusEPA and vitamin E, the magnitude of the decrease being higher with the combined supplement $(65.5 \%$ v. $57.1 \%)$. The TAOC is improved only by dietary supplementation with vitamin E $(17.2 \%)$.

1. Djazayery A \& Jazayery S (2008) In Wild-Type Food in Health Promotion and Disease Prevention, pp. 309-321 [F De Meester and RR Watson, editors]. Totowa, NJ: Humana Press.

2. Venkatraman JT, Leddy J \& Pendergast D (2000) Med Sci Sports Exerc 32, Suppl., S389-S395. 